\title{
Bestimmung der funktionellen Gruppen von Huminstoffen
}

Von F. Martin*), P. Dubach, N. C. Mebta und H.Deuel $\dagger$

Aus dem Agrikulturchemischen Institut der Eidgenössischen Technischen Hochschule, Zürich

(Eingegangen: 30.5. 1963)

Einleitung

Mit der Analyse der funktionellen Gruppen von Huminstoffen aus Böden haben sich nur wenige Forscher beschäftigt. In der Literatur finden sich viele Hinweise, jedoch wenige Beweise für das Vorkommen fast aller sauerstoffhaltigen Gruppen (Karboxylgruppen; chinoide-, phenolische-, enolische- und alkoholische Hydroxylgruppen; Karbonylgruppen und Athergruppen). Es bestehen nicht nur Meinungsverschiedenheiten über die Menge der einzelnen Gruppen, sondern auch über deren Existenz. Weil für Huminstoffe praktisch keine Reinheitskriterien bestehen, sind größere Unstimmigkeiten zu erwarten, besonders, wenn Huminstoffe verschiedener Böden untersucht werden. Allerdings wurden neulich auch bei der Analyse sehr ähnlicher Fulvosäurepräparate durch verschiedene Forscher $(29,36)$ völlig andere Resultate erzielt. Während im einen Fall 9 mäq Karboxylgruppen pro g bestimmt wurden $(35,36)$, führte die Analyse im anderen Fall zu Zweifeln an der Existenz von Karboxylgruppen (29). Schwierigkeiten müssen deshalb auch auf die Natur der Huminstoffe zurückzuführen sein: hochmolekularer Charakter (28) (unvollständige Reaktionen, Adsorption von Reagenzien); extreme Polydispersität (unerwünschte Fraktionierungen beim Aufarbeiten von Reaktionsgemischen); Empfindlichkeit $(28 ; 31$, S. 58-61) gegenüber Alkali sowie Alkali und Säure bei erhöhter Temperatur (verunmöglicht viele Reaktionen, z. B. quantitative Verseifung); Nebeneinander vieler und offenbar verschiedener Gruppen (verändert die Reaktivität der Gruppen, vermindert die Spezifität der Reagenzien).

Die meisten Untersuchungen galten der Säurenatur der Huminstoffe. Dazu wurden die folgenden Methoden angewendet: Titrationen im wäßrigen und nicht wäßrigen Milieu (10,11,29,30,36), Titrationen in Gegenwart mehrwertiger Kationen (Studium der Chelatbildung) (3, 20, 26), Reaktion mit *) Dipl.-Chem. F. Martin, Universitätsstraße 2, Züridh/Sdhweiz. 
$\mathrm{Ba}(\mathrm{OH})_{2}$ (für totale Azidität) $(33,36)$, Reaktion mit Ca-Azetat und $\mathrm{KI} / \mathrm{KIO}_{3}$ sowie Dekarboxylierung (für Karboxylgruppen) $(33,36)$, Reaktion mit Azetanhydrid und Dinitrofluorbenzol (für Hydroxylgruppen) 14, 16, 19, 29, 36), Methylierungen und Verseifungen (Unterscheidung von Karboxyl- und phenolischen und alkoholischen Hydroxylgruppen (4, 18, 19, 21). Die Azidität der Huminstoffe wurde in der Folge allen möglichen aziden Gruppen zugeschrieben: Karboxyl- (4), Hydroxychinon- (14, 24), phenolischen Hydroxyl- (24, 29), und enolischen Hydroxylgruppen $(19,29)$. Da sich die $\mathrm{pK}$-Werte dieser Gruppen überschneiden können - 2,5-Dihydroxychinon ist eine stärkere Säure als Essigsäure (14) - ist eine genaue Unterscheidung mit den aufgezählten Methoden, welche mit Ausnahme der unspezifischen Dekarboxylierung, pK-abhängig sind, nicht möglich. Hinzu kommt, daß auch die pK-Werte individueller Gruppen, wegen dem polybasischen Aziditäts-Charakter der Huminstoffe, verschieden sein müssen (11). Dies sollte auch berücksichtigt werden bei allen Versuchen und Uberlegungen über die Fähigkeit der Huminstoffe, mehrwertige Kationen stark, evtl. als Chelate, zu binden (31, S. 77-81, 20). Es wurde nämlich gezeigt, daß z. B. Polyacrylsäure $\mathrm{Cu}^{++}$stärker bindet als die analoge monomere Essigsäure und Glutarsäure (10).

Zuerst muß also die Anwesenheit oder Abwesenheit individueller azider Gruppen mit vom pK-Wert unabhängigen Methoden gezeigt werden. Dies gelang bis jetzt einzig für Karboxylgruppen $(13,23,32)$, mit Hilfe der IRSpektroskopie (Verschiebung der Karbonylabsorption bei der Salzbildung). Die wiederholt beobachtete reversible Aufhellung von Huminstoff-Lösungen bei Behandlung mit Reduktionsmitteln $(13,37)$ könnte auf chinoiden Charakter weisen, welcher allerdings durch reduktive Azetylierung nicht bestätigt werden konnte $(13,32)$. Bei der Bestimmung der Karbonylgruppen in Huminstoffen mit Hydroxylamin und Dinitrophenylhydrazin wurden unerwartet kleine Werte (um 1-2 mäq/g) erhalten $(14,29,36)$; Reaktion mit $\mathrm{NaBH}_{4}$ (29) hingegen ergab unwahrscheinlich hohe Werte (14 mäq/g). Beim Lignin konnte diese Unstimmigkeit mit der Anwesenheit von Schwermetallen erklärt und durch Ausschluß des Luftsauerstoffs behoben werden (27). Huminstoffe lassen sich halogenieren, was auf aromatische oder andere ungesättigte Strukturen weist. Wegen der leichten Oxydierbarkeit der Huminstoffe ließen sich die Doppelbindungen nicht durch Bromanlagerung bestimmen $(9,29)$.

Das neuartige Reduktionsmittel Diboran schien, wegen seiner besonderen Reaktionsweise mit fast allen sauerstoffhaltigen (und auch anderen) Gruppen und Doppelbindungen sowie wegen seiner geringen Molekülgröße (keine sterische Hinderung) geeignet, die Analyse der funktionellen Gruppen von Huminstoffen auf anderer Basis und unabhängig von pK-Werten zu ermöglichen. Dieses Reagens wurde bis jetzt hauptsächlich für präparative und weniger für analytische $\mathrm{Z}$ wecke verwendet $(5,6)$.

Diboran reagiert in inerten Lösungsmitteln (z. B. Tetrahydrofuran $=$ THF) mit den hier interessierenden Gruppen organischer Verbindungen in folgender 
Weise: Karboxyl- und Hydroxylgruppen entwickeln sofort je ein Mol Wasserstoff pro Gruppe, welcher als aktiver Wasserstoff gemessen werden kann. Karboxylgruppen sowie Karbonylgruppen (keine $\mathrm{H}_{2}$-Entwicklung) werden in dieser Reihenfolge sehr rasch zu den entsprechenden Alkoholgruppen reduziert. Ester und Laktone werden (ohne $\mathrm{H}_{2}$-Entwicklung) nur sehr langsam zu den entsprechenden Alkoholen reduziert. Nichtaromatische Doppelbindungen und Dreifachbindungen werden sehr rasch hydroboriert, d. h. es wird $\mathrm{H}$ und $\mathrm{B}$ angelagert, wobei die entstehende C-B-Bindung gegen Wasser stabil ist.

In dieser Arbeit wird über die Analyse der sauerstoffhaltigen Gruppen von Huminstoffen berichtet. Karboxylgruppen wurden pK-unabhängig mit Diboran, Hydroxylgruppen durch Azetylierung und Karbonylgruppen durch Reaktion mit $\mathrm{NaBH}_{4}$ bestimmt. An einer THF-löslichen Fulvosäure wurde die Methodik eingehend geprüft und dann bei einer Reihe von Fraktionen verschiedenen mittleren Molekulargewichtes angewendet.

\section{Material}

Für die Untersuchungen wurden ausschließlich Huminstoffe aus dem $\mathrm{B}_{\mathrm{h}}$-Horizont eines Podsols (Nante) verwendet. Die Gewinnung der THF-löslichen Fulvosäure FS-2 sowie der Braunbuminsäure B-HS ist in einer früheren Arbeit beschrieben worden (28). Aus der Gesamt-Fulvosäurefraktion (FS- $\mathrm{HCl}$ ), mit $1 \mathrm{~N} \mathrm{HCl}$ extrahiert und über das Fe-Salz isoliert (12), wurden durch fraktionierte Dialyse die Fulvosäurefraktionen $D_{1}$

\section{Tabelle 1}

Fraktionierte Dialyse der Fulvosäurc FS-HCl (20 g)

Fractional dialysis of the fulvic acid FS- $\mathrm{HCl}(20 \mathrm{~g})$

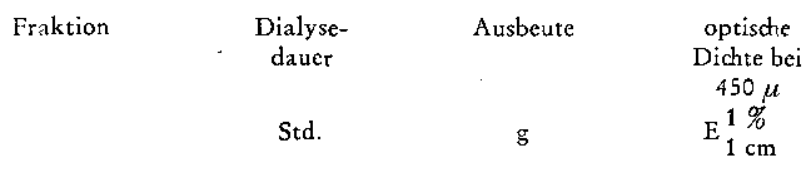

$\begin{array}{lrrr}\mathrm{D}_{1} & 1 & 0,772 & 9,5 \\ \mathrm{D}_{2} & 16 & 3,709 & 10,0 \\ \mathrm{D}_{3} & 19 & 0,623 & 10,3 \\ \mathrm{D}_{4} & 65 & 5,220 & 11,7 \\ \mathrm{D}_{5} & 73 & 0,508 & 13,2 \\ \mathrm{D}_{6} & 88 & 0,800 & 13,8 \\ \mathrm{D}_{7} & 121 & 1,137 & 16,6 \\ \mathrm{D}_{8} & 137 & 0,761 & 18,3 \\ \mathrm{O}_{9} & 184 & 0,637 & 18,4 \\ \mathrm{D}_{10} & 328 & 1,009 & 19,9 \\ \mathrm{D}_{11} & 383 & 0,924 & 23,0 \\ \mathrm{D}_{12} & 472 & 0,556 & 25,4 \\ \mathrm{D}_{13} & \text { Rüdsstand } & 2,202 & 28,5 \\ \text { FS-2 } & & & \\ \mathrm{B}-\mathrm{HS} & - & - & 20,0 \\ & - & - & 64,0\end{array}$


bis $D_{13}$ gewonnen. $20 \mathrm{~g}$ von FS- $\mathrm{HCl}(0,9 \%$ Asche, $0,8 \% \mathrm{~N})$ wurden in $150 \mathrm{ml}$ Wasser gelöst, in einen Zellophanschlauch gegeben und in einem 2-Liter-Meßzylinder gegen Wasser dialysiert. Nach verschiedenen Zeiten wurden die herausdialysierten Fulvosäuren unter Vakuum konzentriert, gefriergetrocknet, gewogen und die optische Dichte einer Lösung von $5-20 \mathrm{mg}$ in $100 \mathrm{ml} \mathrm{NaOH}$-Glycinpuffer (28) gemessen (Tabelle 1).

\section{Methoden}

1. Die IR-Spektren wurden im Laboratorium von Herrn Dr. Simon (Laboratorium für organische Chemie, E.T.H.) aufgenommen. Die untersuchten Na-Salze wurden durch Titration bis $\mathrm{pH} 10$ gebildet und die Lösung gefriergetrocknet.

2. Die Elementaranalysen, die Acetylbestimmungen sowie die Bestimmung des aktiven Wasserstoffs nach Zerewitinoff wurde im mikroanalytischen Laboratorium von Dr. A. Bernhardt, Max-Planck-Institut für Kohlenforschung, Mülheim (Ruhr) ausgeführt.

3. Herstellung von Diboran: Eine $1 \mathrm{M}$ Lösung von $\mathrm{NaBH}_{4}$ in Diäthylenglykoldimethyläther $(7,56 \mathrm{~g} / 200 \mathrm{ml})$ wurde langsam $\mathrm{zu}^{\mathrm{B}} \mathrm{BF}_{\mathrm{s}}-\mathrm{K}$ thylätherat $(50 \mathrm{ml})$ tropfen gelassen, und das entwickelte Diboran-Gas wurde im $\mathrm{N}_{2}$-Strom im THF $(120 \mathrm{ml})$ geleitet (5). Die erhaltene $1 \mathrm{M}$ Lösung von Diboran $\left(\mathrm{B}_{2} \mathrm{H}_{6}\right)$ in THF wurde im Kühlschrank unter $\mathrm{N}_{2}$ aufbewahrt und war 2 Monate haltbar.

4. Reduktion der Fulvosäure FS-2: In drei Kolben wurden je $50 \mathrm{mg}$ von FS-2 in $5 \mathrm{ml}$ trockenem THF gelöst und $5 \mathrm{ml}$ Diboranlösung zugegeben. Die Kolben wurden mit einem Polyäthylenstopfen mit Chlorkalziumrohr geschlossen und in einem Exsikkator mit $\mathrm{P}_{2} \mathrm{O}_{5}$ im Dunkeln bei Zimmertemperatur aufbewahrt. Nach zwei Tagen wurde die Reaktionslösung des ersten Kolbens am Vakuum eingedampft. Der Rückstand war nicht mehr so gut wasserlöslich wie FS-2. Die Borsäure wurde durch wiederholtes Lösen in Methanol, Versetzen mit etwas $\mathrm{CH}_{2} \mathrm{~N}_{2}$ und Eindampfen als Methylester entfernt. Das IR-Spektrum in KBR zeigte nur eine sehr geringe Verminderung der Absorption im Karbonylgebiet. Nach einer Wodhe bzw. nach einem Monat wurden die Reaktionslösungen der beiden anderen Kolben ebenfalls eingedampft; die reduzierten Fulvosäuren lösten sich nicht mehr in Wasser. Die Borsäure wurde deshalb durch Dialyse gegen Wasser (1 Woche) entfernt. Die Lösung wurde gefriergetrocknet und die Präparate sowie ihre bei $\mathrm{pH} 10$ gebildeten $\mathrm{Na}$-Salze IR-spektroskopisch untersucht. Ein größerer Ansatz von $500 \mathrm{mg}$ FS-2 wurde während eines Monats vollständig reduziert (= FS-2 R, Ausbeute $60 \%$, Verlust niedrigmolekularer Fraktionen bei der Dialyse).

5. Bestimmung des aktiven Wasserstoffs mit Diboran: Die Substanz wurde in ein gut getrocknetes Schliffkölbchen $(75 \mathrm{ml})$ mit flachem Boden und seitlichem Rohransatz eingewogen, mit einer trockenen Pipette $5 \mathrm{ml}$ trockenes THF zugegeben, der Hauptschliff mit einer Gummikappe gut verschlossen und mit dem seitlichen Rohransatz über Kugelschliff-Verbindung an eine Gasbürette von $100 \mathrm{ml}$ angeschlossen. Als Absperrflüssigkeit diente Wasser. Mit einer Medizinalspritze wurde $1 \mathrm{ml}$ Diboranlösung durch die Gummikappe sorgfältig in den Kolben gespritzt. Die Wasserstoffentwicklung war bei Substanzen, die sich in THF lösten, nach 1-2 Minuten beendet. Nach genau 5 Minuten wurde der Wasserstoff in der Gasbürette mit Hilfe eines Niveaugefäßes unter Atmosphärendruck gemessen und die Temperatur und der Barometerstand notiert. Es wurden jeweils mindestens drei Bestimmungen mit verschiedenen Einwagen, welche ca. 30,40 und $50 \mathrm{ml} \mathrm{H}_{2}$ entwickelten, durchgeführt. Jedesmal wurde graphisch ge- 
prüft, ob die $\mathrm{H}_{2}$-Entwicklung sich linear zur Einwaage verhält, und dann durch Extrapolation auf Einwaage Null der Blindwert der $\mathrm{H}_{2}$-Entwicklung ermittelt. Die zu prüfenden Substanzen müssen vor der Analyse gut getrocknet werden (1 $\mathrm{Mol} \mathrm{H}_{2} \mathrm{O}$ gibt 2 Mol aktiven Wasserstoff). Da Huminstoffe im allgemeinen stark hygroskopisch sind und nach Trodknung nicht mehr gut in Lösung gehen, wurden sie luftfeucht analysiert und das Resultat für Wasser korrigiert. Der Wassergehalt wurde durch Trodknung unter Hochvakuum bei $40^{\circ} \mathrm{C}$ über $\mathrm{P}_{2} \mathrm{O}_{5}$ bestimmt.

Für die Fulvosäurefraktion $\mathrm{D}_{2}$ z. B. gestaltete sich die Bestimmung wie folgt:

Einwaagen: 20,35 und $50 \mathrm{mg}$. $\mathrm{H}_{2}$-Entwicklung bei $23^{\circ} \mathrm{C}$ und $732 \mathrm{~mm} \mathrm{Hg}: 31,0 \mathrm{ml}$, $38,75 \mathrm{ml}$ und 46,5 ml. Blindwert: $20,7 \mathrm{ml}$. Wassergehalt: 7,6\%. $-\mathrm{H}_{2}$-Entwidklung von $1 \mathrm{~g}$ luftfeuchter Substanz für Normalbedingungen korrigiert: $459 \mathrm{ml} . \mathrm{H}_{2}$-Entwicklung von Wasser in $1 \mathrm{~g}$ luftfeuchter Substanz bei Normalbedingungen: $189 \mathrm{ml} . \mathrm{H}_{2}$-Entwicklung $1 \mathrm{~g}$ trockener Substanz bei Normalbedingungen: $292,5 \mathrm{ml}$, entsprechen $13 \mathrm{mäq}$ aktivem Wasserstoff.

In THF unlösliche Substanzen $\left(\mathrm{D}_{10}, \mathrm{D}_{13}, \mathrm{~B}-\mathrm{HS}\right)$ wurden in $4 \mathrm{ml}$ trodkenem Dimethylformamid gelöst, 1,5 $\mathrm{ml}$ Diboran-Lösung zugespritzt und im übrigen wie vorher beschrieben verfahren.

Von allen geprüften Modellsubstanzen (Hydroxyl- und Methoxylsubstituierte Benzoeund Benzoldikarbonsäuren, Polyphenole, substituierte Chinone und andere hydroxylierte oder karboxylierte aromatische Verbindungen) wurde eine der Theorie entsprechende $\mathrm{H}_{2}$-Entwicklung gemessen.

6. Bestimmung der Karbonylgruppen mit $\mathrm{NaBH}_{4}$ (27): Je $50 \mathrm{mg}$ Substanz wurde in den unter 5. beschriebenen Reaktionskolben gegeben, in $2 \mathrm{ml}$ Wasser gelöst und mit $2 \mathrm{~N} \mathrm{NaOH}$ pH 7-8 eingestellt. Dann wurden $3 \mathrm{ml}$ einer alkoholischen Lösung von Borsäure $(1 \mathrm{~g} / 100 \mathrm{ml})$ zugegeben. Der Kolben wurde mit der Gummikappe geschlossen und während 2 Stunden durch eine Impfnadel Argon in die Lösung eingeleitet um den Luftsauerstoff zu entfernen. Der Kolben wurde, wie unter 5 . beschrieben, an die Gasbürette geschlossen und $3 \mathrm{ml} \mathrm{NaBH}$-Lösung ( $1 \mathrm{~g} / 100 \mathrm{ml} 0,1 \mathrm{~N} \mathrm{NaOH}$ ) eingespritzt. Nach 3 Stunden wurden $2 \mathrm{ml} 2 \mathrm{~N} \mathrm{H}_{2} \mathrm{SO}_{4}$ eingespritzt und die $\mathrm{H}_{2}$-Entwicklung gemessen. Gleichzeitig wurde ein Blindversuch auf genau gleiche Weise durchgeführt. Aus der Differenz ergab sich der für die Reduktion der Karbonylgruppen verbrauchte Wasserstoff (1 Mol $\mathrm{H}_{2} /$ mäq Karbonyl). - Die Methode ergab mit p-Benzochinon und Morin der Theorie entsprechende Werte.

7. Azetylierung: Je $20 \mathrm{mg}$ Huminstoff wurden in $10 \mathrm{ml}$ Azetanhydrid während 24 Stunden bei $60^{\circ} \mathrm{C}$ im Olbad erwärmt. (Bei FS-2-R, B-HS, $D_{10}$ und $D_{13}$ wurden zwei Tropfen konc. $\mathrm{H}_{2} \mathrm{SO}_{4}$ zugegeben.) Die braune Lösung wurde in Eiswasser gegossen und 6 Stunden im Kühlschrank stehen gelassen. Das ausgefällte Azetat wurde auf einer G-4-Nutsche abgenutscht, mit kaltem Wasser gut gewaschen und mit Aceton aus der Nutsche gelöst. Die Lösung wurde konzentriert und das Azetat in Petroläther ausgefällt. Die Azetylgehalte waren wie folgt:

FS-2 $=13,3 \%$, FS-2-R $=31,0 \%, \quad D_{2}=7,8 \%, \quad D_{4}=12,7 \%, D_{7}=14,34 \%$, $\mathrm{D}_{10}=15,9 \%, \mathrm{D}_{13}=19,6 \%, \mathrm{~B}-\mathrm{HS}=15,6 \%$.

8. Gesamtazidität durch Reaktion mit $\mathrm{Ba}(\mathrm{OH})_{2}(33,36): 50 \mathrm{mg}$ Huminstoff wurden in ein Zentrifugenglas $(250 \mathrm{ml}$ ) eingewogen, in $10 \mathrm{ml}$ Wasser gelöst, bzw. suspendiert,

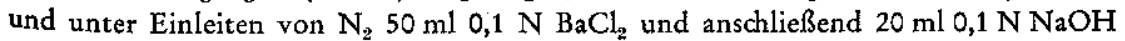


zugegeben. Das Zentrifugenglas wurde geschlossen, 16 Stunden geschüttelt und dann zentrifugiert. Aliquote der überstehenden, nur schwach gefärbten Lösung wurden mit $0,1 \mathrm{~N} \mathrm{HCl}$ und Phenolphthalein titriert. Die Gesamtazidität ergab sich nach der Titration eines Blindversuches.

9. "Karboxylgebalt" durcb Reaktion mit Ba-Azetat: $50 \mathrm{mg}$ Huminstoff wurden in einem Zentrifugenglas wie unter 8 . beschrieben gelöst. Unter $\mathrm{N}_{2}$ wurden $50 \mathrm{ml} 3 \mathrm{~N}$ Ba-Azetat (ergab besseren Niederschlag mit Huminstoffen als Ca-Azetat) zugegeben, 16 Stunden geschüittelt und zentrifugiert. Aliquote der überstehenden, fast farblosen Lösung wurden mit $0,1 \mathrm{~N} \mathrm{NaOH}$ (Phenolphthalein) titriert. Die verbrauchte Lauge ergab, da der Blindwert Null war, direkt den „Karboxylgehalt“.

\section{Resultate und Diskussion}

Abbildung 1 zeigt die IR-Spektren der Fulvosäurefraktion FS-2 (Kurve 1) und des bei pH 10 gebildeten Na-Salzes (Kurve 2). Ein gleiches Spektrum zeigt das Al-Salz, welches durch Lösen von FS-2 in Wasser, Ausfällung mit $1 \mathrm{~N} \mathrm{AlCl}_{3}$ (bei leicht saurer Reaktion), Dialyse und Gefriertrocknung erhalten wurde. Sie bestätigen auch für dieses Huminstoffpräparat die oben erwähnte Verschiebung des Hauptteils der Karbonylabsorptionsbande von 5,7-5,8 $\mu$ nach $6,1-6,2 \mu$, was auf die Anwesenheit einer bedeutenden Menge von Karboxylgruppen weist. Das Hauptziel der Untersuchungen bestand deshalb in einer $\mathrm{pK}$-unabhängigen Bestimmung der Karboxylgruppen.

Diboran reduziert Karboxylgruppen normalerweise sehr rasch zu primären Alkoholgruppen. Die Bestimmung der Karboxylgruppen sollte sich deshalb 1) aus dem verbrauchten Hydrid (6), 2) aus der Elementaranalyse des reduzierten Produkts (Zunahme des Wasserstoffs und Abnahme des Sauerstoffs) und 3) aus der Zunahme der Hydroxylgruppen ergeben, wobei das Resultat für reduzierte Karbonylgruppen korrigiert werden müßte.

Die restlose Reduktion der Karboxylgruppen wurde IR-spektroskopisch an der reduzierten Fulvosäure und ihrem Na-Salz überprüft. Die Reduktion der Karboxylgruppen erfolgte viel langsamer als in der Literatur für einfache organische Säuren beschrieben ist (6). Nach 2 Tagen war das IR-Spektrum kaum verändert, nach einer Woche (Abb. 1, Kurve 3) war die Karbonylabsorptionsbande bei 5,7-5,8 $\mu$ deutlich kleiner geworden, und nach einem Monat (Kurve 4) war in diesem Gebiet keine Absorptionsbande mehr festzustellen. Hingegen erstreckte sich von $5,6-6,0 \mu$ immer noch eine diffuse Restabsorption, welche nach Salzbildung nicht verändert wurde und deshalb nicht von Karbonylgruppen stammt.

Die Messung des Hydrid-Verbrauchs war technisch nicht möglich, nachdem zur vollständigen Reduktion eine sehr lange Reaktionszeit benötigt wurde. Es wurde deshalb das Ausgangsmaterial und das vollständig reduzierte Produkt analysiert (Tab. 2). Da bei der Reduktion der Kohlenstoff nicht verändert wird, müssen die Analysen auf gleiche Kohlenstoffmengen umgerechnet werden 


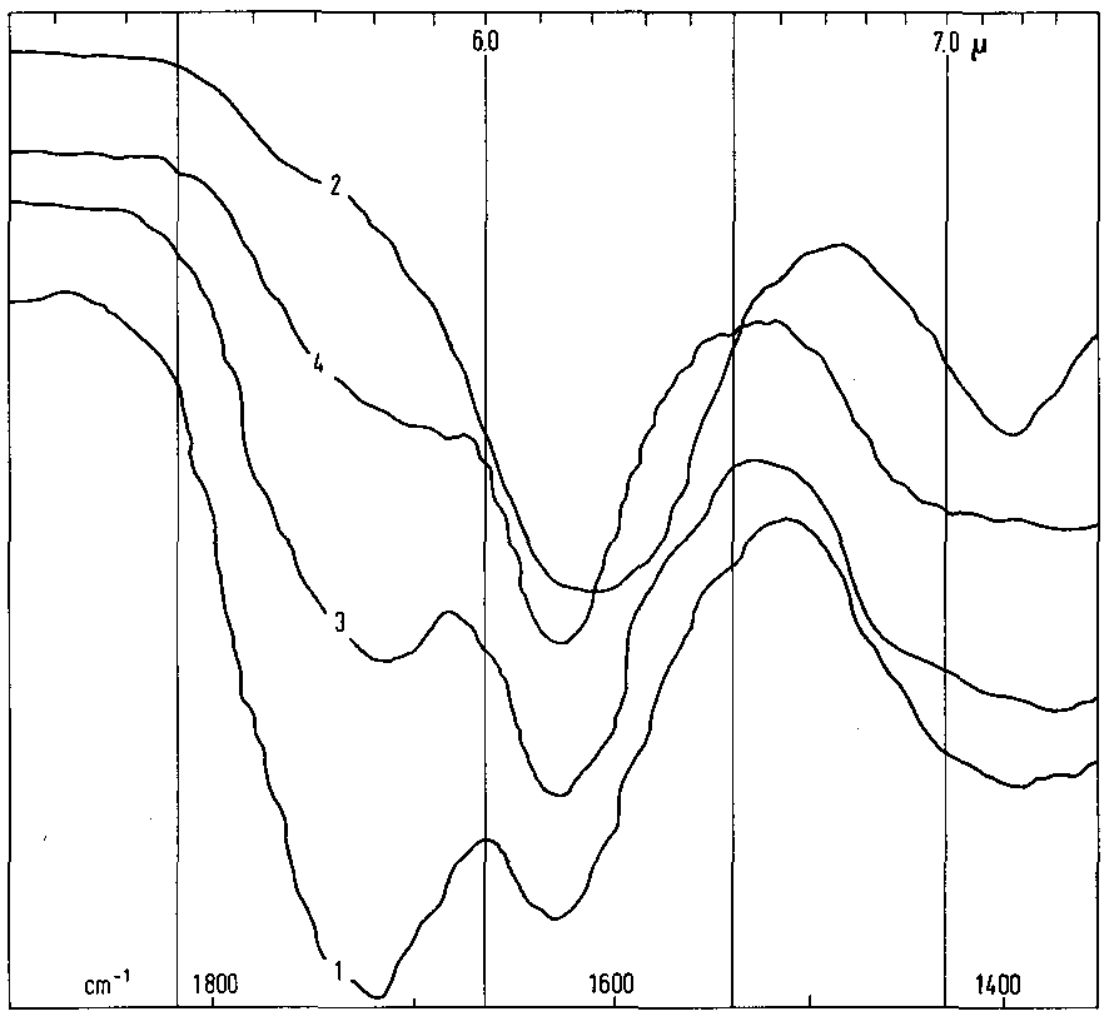

Abbildung 1

IR-Spektren der Fulvosäure FS-2 und Derivaten

Kurve 1: FS-2; Kurve 2: Na-Salz von FS-2

Kurve 3: FS-2 nach 1 Woche Reduktion mit Diboran

Kurve 4: FS-2 nach 1 Monat Reduktion mit Diboran

IR-spectra of fulvic acid FS-2 and derivatives

Curve 1: FS-2; curve 2: Na-salt of FS-2

curve 3: FS-2 after reduction with diborane for 1 weck

curve 4: FS-2 after reduction with diborane for 1 month

(FS-2-R*). Der Wasserstoff nahm durch die Reduktion um $15 \mathrm{mäq} / \mathrm{g}$ zu und der Sauerstoff um 7,5 mäq/g ab, was einem Karboxylgehalt von 7,5 mäq/g entsprechen würde. Die azetylierbaren Hydroxylgruppen nahmen um 5,8 mäq/g zu. Im reduzierten Präparat wurden mit $\mathrm{NaBH}_{4}$ keine Karbonyle mehr festgestellt. Nach der Korrektur für reduzierte Karbonyle (1 mäq/g) erhält man für den Karboxylgehalt aus der Wasserstoff zunahme 6,5 mäq/g, aus der Sauerstoffabnahme 7,4 mäq/g und aus der Azetylzunahme 4,8 mäq/g. Wegen möglicher nicht vollständiger Azetylierung bzw. Elimination von Nichtkarboxyl- 
Sauerstoff (2), ist 4,8 mäq/g als unterer und 7,5 mäq/g als oberer Grenzwert der unabhängig von $\mathrm{pK}$ bestimmten Karboxylgruppen anzusehen.

Durch Titration mit $\mathrm{NaOH}$ bis $\mathrm{pH} 8,5$ wurden 5,8 mäq und durch Reaktion mit Ba-Azetat 7,8 mäq/g saure Gruppen bestimmt. Nach Berücksichtigung der durch Reduktion mit Diboran bestimmten Karboxylgruppen bleibt deshalb kaum Platz für stark saure Hydroxychinone oder Enole. Das reduzierte Präparat enthielt kein Bor, was zeigt, daß die Fulvosäure keine hydroborierbaren, olefinischen Doppelbindungen, z. B. Enole (7), enthält.

Die reduzierte Fulvosäure war im Unterschied zum Ausgangsmaterial in allen gebräuchlichen Lösungsmitteln wie Wasser, Säuren, Alkohol, Dioxan, Dimethylformamid, THF und Benzol unlöslich; sie ging erst in Boratpuffer von $\mathrm{pH} 11$ in Lösung.

Azetylierung in Azetanhydrid bei $60^{\circ} \mathrm{C}$ und mikroanalytische Bestimmung des Azetylgehaltes durch Verseifung ergab 3,6 mäq/g Hydroxylgruppen (Tab. 2). Durch Azetylierung und titrimetrische Bestimmung des unverbrauchten Azetanhydrids $(29,36)$ wurden viel größere Werte erhalten (7-9 mäq/g). Diese Methode scheint, wegen des sauren Charakters der Huminstoffe, zweifelhafte Werte zu geben (25).

Für einen geringen Gehalt an Hydroxylgruppen sprechen auch Oxydationsversuche mit $\mathrm{NaIO}_{4}$ partiell methylierter Huminstoffe (FS-2, B-HS). Bei der $\mathrm{NaIO}_{4}$-Oxydation der Monomethyläther von o- und $\mathrm{p}-\mathrm{Di}$ hydroxybenzolen wird Methanol abgespalten (1). Methylierung der Modellsubstanzen Brenzkatechin und $\mathrm{Hy}$ drochinon mit $\mathrm{CH}_{2} \mathrm{~N}_{2}$ ergab vorwiegend Monoäther- und erst nach längerer Reaktionszeit auch Dimethyläther-Derivate. Deshalb sollte man ound p-Dihydroxybenzol-Strukturen in Huminstoffen nach partieller Methylierung und Oxydation mit $\mathrm{NaIO}_{4}$ 
auf Grund der Methanol-Produktion bestimmen können. Die partiell methylierten Huminstoffe ergaben aber bei der Oxydation gleich viel Methanol wie Blindversuche mit Wasser allein, was lediglich auf eine Verseifung von Methylestergruppen deutet.

Nach Zerewitinoff wurden $6 \mathrm{mäq} / \mathrm{g}$ aktiven Wasserstoffs bestimmt. Dieser Wert ist viel zu tief, denn die bestimmten Karboxyl- und Hydroxylgruppen verlangen einen Wert von 8,4 bis $11,1 \mathrm{mäq} / \mathrm{g}$. Dic Methode nach Zerewitinoff ergibt nicht immer stöchiometrische Werte (34). Mit Diboran konnte bei einer großen Zahl von Verbindungen der aktive Wasserstoff (von Karboxyl- und Hydroxylgruppen genau bestimmt werden (s. unter Methoden und [25]). Die Bestimmung des aktiven Wasserstoffs der Fulvosäure mit Diboran ergab $11,6 \mathrm{mäq} / \mathrm{g}$. Dieser Wert stimmt mit der oberen Grenze der Summe der bestimmten Karboxyl- und Hydroxylgruppen $(8,4-11,1 \mathrm{mäq} / \mathrm{g})$ gut überein. Es ist deshalb möglich, Karboxylgruppen in Huminstoffen aus dem Unterschied zwischen dem aktiven Wasserstoff (Diboran) und den Hydroxylgruppen (Azetylierung) zu errechnen.

Die Bestimmung der Gesamtazidität mit $\mathrm{Ba}(\mathrm{OH})_{2}$ ergab 11,9 mäq/g. Dieser Wert stimmt gut mit dem aktiven Wasserstoff überein und weist auf die Abwesenheit nicht azider, d. h. alkoholischer Hydroxylgruppen.

Durch Reaktion mit $\mathrm{NaBH}_{4}$ (nach sorgfältigem Ausschluß des Sauerstoffs) wurde ein Karbonylgehalt von $1 \mathrm{mäq} / \mathrm{g}$ bestimmt (Tab. 2). Damit wurden in 4,8-7,5 mäq Karboxyl-, in 3,6 mäq Hydroxyl- und in 1 mäq Karbonylgruppen 15,2-19,6 mäq oder 55-70\% des Sauerstoffs $(27,5$ mäq/g) der Fulvosäure erfaßt. Es bleiben $30-45 \%$ oder 7,9-12,3 mäq/g Rest-O, was auf einen beträchtlichen Gehalt an Äther-O deutet.

Es war nun interessant, eine Reihe verschiedener Huminstoff-Präparate zu analysieren. Die Fulvosäurepräparate $D_{1}$ bis $D_{13}$ wurden durch fraktionierte Dialyse und die Braunhuminsäure B-HS durch Fraktionierung mit $\mathrm{NaCl}$ erhalten. Gelfiltration (28) ergab für die Fulvosäurefraktionen $D_{2}$ bis $D_{13}$ und für die Braunhuminsäure B-HS (28) ein zunehmend größeres mittleres Molekulargewicht (Abb. 2), FS-2 hatte eine ähnliche Molekulargewichtsverteilung wie $\mathrm{D}_{7}$ (28).

Wie aus Tab. 3 hervorgeht, nahm der aktive Wasserstoff mit steigendem Molekulargewicht von 13,0 mäq/g $\left(\mathrm{D}_{2}\right)$ auf 7,1 mäq/g (B-HS) ab und stimmte ziemlich gut mit der Gesamtazidität $\left(\mathrm{Ba}(\mathrm{OH})_{2}\right)$ überein. Die azetylierbaren Hydroxylgruppen stiegen in den Dialysefraktionen mit dem Molekulargewicht und waren in B-HS wieder etwas kleiner. Der Karboxylgehalt, errechnet aus dem Unterschied zwischen dem aktiven Wasserstoff und den Hydroxylgruppen sowie der Karboxylgehalt nach der Ba-Azetat-Methode nahmen mit steigendem Molekulargewicht der Fraktion stark ab. Der Unterschied zwischen den beiden Methoden stieg mit zunehmendem Molekulargewicht und Hydroxylgehalt der Fraktionen, vermutlich wegen der Reaktion von Hydroxylgruppen mit $\mathrm{Ba}$ 


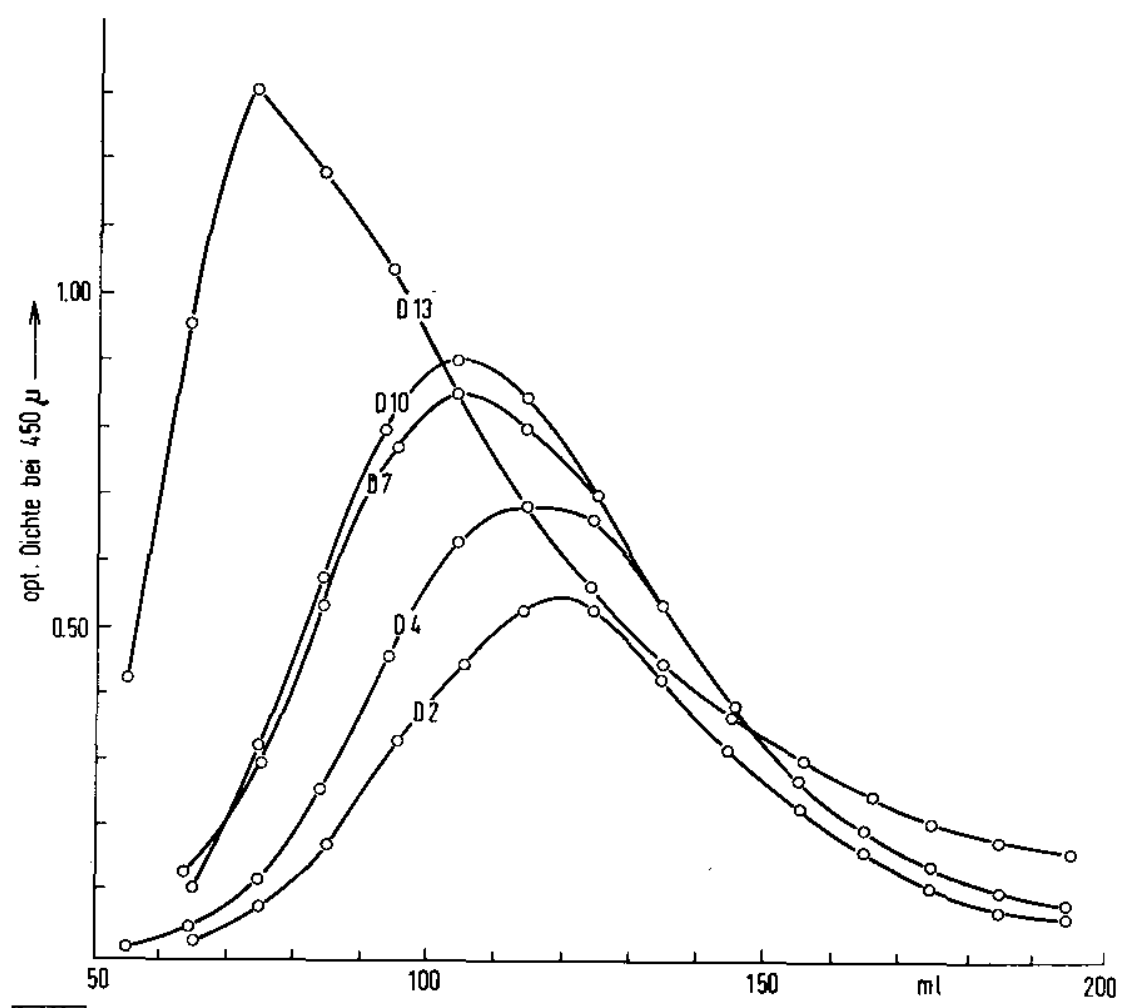

P2966.2

Abbildung 2

Molekulargewichtsverteilung der Fulvosäurefraktionen $D_{2}, D_{4}, D_{7}, D_{10}$ und $D_{13}$ an Sephadex $G-75$

Molecular weight distribution of fulvic acid fractions $D_{2}, D_{4}, D_{7}, D_{10}$ and $D_{13}$ on Scphadex G-75

(Komplexbildung?). Der Karbonylgehalt der Fraktionen ist ziemlich konstant (1,9-2,5 mäq/g) mit Ausnahme von Fraktion FS-2, welche einen geringeren Karbonylgehalt (1 mäq/g) hat.

Für alle Fraktionen wurde der Rest-O oder Skelett-O (Tab. 3, Kolonne 8) durch Subtraktion des Sauerstoffs der bestimmten funktionellen Gruppen vom Gesamtsauerstoff (Kolonne 7) in mäq/g ausgerechnet. Er stieg in den Fulvosäurefraktionen mit steigendem Molekulargewicht von 3,5 mäq/g auf $13,4 \mathrm{mäq} / \mathrm{g}$ und war in B-HS wieder etwas kleiner. In $\mathrm{D}_{13}$ waren ca. 50\% des Sauerstoffs nicht in funktionellen Gruppen erfaßt worden. $\mathrm{Da}$ die verschiedenen Huminstoff-Fraktionen unterschiedliche Mengen funktioneller Gruppen enthalten, lassen sie sich nicht direkt vergleichen. Erst das nach Abzug der funktionellen 


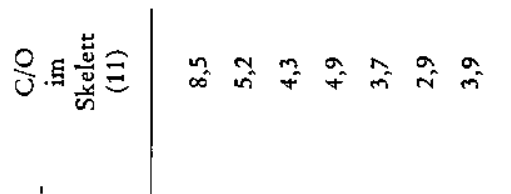

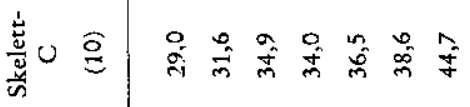

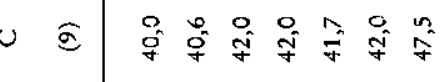

$\stackrel{20}{-10}$

䒠

คำ

tis

:

Fi

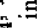

คำ

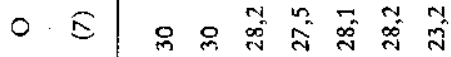

थि थै

Gruppen (COOH für Karboxyl, $\mathrm{OH}$ für $\mathrm{Hy}$ droxyl und $O$ für Karbonyl) erhaltene „Huminstoff-Skelett" gestattet den richtigen Vergleich. Auf Grund der Abbauversuche (8) muß man sich dieses Skelett aus aromatischen Bausteinen vorstellen. Das Problem, den Rest- oder Skelett-O in einem solchen Skelett als Ather-O zu betrachten, kommt im $\mathrm{C} / \mathrm{O}$-Verhältnis des Skeletts von 3-8,5 (Kolonne 11) zum Ausdruck. (Zwei, 3 und 4 Atherbindungen pro Benzolring ergeben ein $\mathrm{C} / \mathrm{O}$-Verhältnis von 6, 4 und 3 ).

In den vorliegenden Huminstoffpräparaten würden demnach bis 4 Atherbindungen pro Benzolring $\left(\mathrm{D}_{13}\right)$ enthalten sein, was unmöglich scheint. Das IR-Spektrum des reduzierten Fulvosäurepräparates FS-2-R (Abb. 2, Kurve 4), welches keine Karboxylgruppen mehr enthält, läßt noch die Anwesenheit von Karbonylgruppen vermuten (Chinone, Laktone?); mit $\mathrm{NaBH}_{4}$ ließen sich allerdings keine bestimmen.

Ahnlich wie bei Kohle ((22) und Lignin $\left(\mathrm{C}_{9}\right.$-Einheit [17]) müssen auch bei Huminstoffen die Analy- 
senresultate statistisch dargestellt werden. Zum Beispiel ließe sich für die Fulvosäure FS-2 (nach Tab. 3) folgende hypothetische, aus einem Benzolring bestehende, monomere $\mathrm{C}_{6}$-Einheit mit 1,4 Karboxyl-, 0,6 Hydroxyl-, 0,18 Karbonylund 1,2 Athergruppen (bzw. 2,4 Ätherbindungen) formulieren.

Bei der Betrachtung des C/O-Verhältnisses im Skelett der verschiedenen untersuchten Huminstoffpräparate ist, neben der Größe des Verhältnisses, dessen Schwankungsbereich von Bedeutung.

In der Frage, ob in Huminstoffen verschiedenen Molekulargewichtes immer ein einheitliches oder ein in bestimmter Richtung sich änderndes Skelett vorliegt, dürfte der Schlüssel zur Erkennung des polymeren Prinzips liegen. Zur genauen Untersuchung dieser Frage müssen die Analysenmethoden aber noch weiter ausgebaut und verfeinert werden.

Diese Arbeit wurde durch Forschungskedite des Schweizerischen Nationalfonds zur Förderung der wissenschafflichen Forschung und des Fonds zur Förderung der Waldund Holzforschung sowie durch ein Austauschstipendium des Consejo Superior de Investigaciones Cientificas de Espana ermöglicht; wir danken bestens für diese Unterstritzung.

\section{Zusammenfassung}

In einer Tetrahydrofuran löslichen Fulvosäurefraktion aus dem $B_{h}$-Horizont eines Podsols wurden die Karboxylgruppen durch Reduktion mit Diboran (4,8-7,5 mäq/g), die Hydroxylgruppen durch präparative Azetylierung (3,6 mäq/g) und die Karbonylgruppen durch Reaktion mit Natriumborhydrid ( 1 mäq/g) bestimmt. Der mit Diboran bestimmte aktive Wasserstoff $(11,6$ mäq/g) stimmte gut mit der Gesamtazidität aus der Reaktion mit Bariumhydroxyd überein (11,9 mäq/g), was gegen die Anwesenheit alkoholischer Hydroxylgruppen spricht. Etwa $60 \%$ des Sauerstoffs fanden sich nicht in den bestimmten funktionellen Gruppen; es ist unwahrscheinlich, daß der gesamte Restsauerstoff in Atherbindungen vorliegt. Bei der Analyse einer Reihe von Huminstoff-Fraktionen mit steigendem Molekulargewicht nahmen die Karboxylgruppen ab, die Hydroxylgruppen zu, die Korbonylgruppen blieben konstant und der Restsauerstoff nahm zu. Eine statistische Darstellung der Analysenresultate mit Hilfe einer hypothetischen monomeren Einheit oder eines Huminstoff-Skeletts, das keine funktionellen Gruppen enthält, wurde diskutiert.

\section{S chrif $\mathrm{tum}$}

(1) Adler, E., and Magnusson, R.: Acta Chem. Scand. 13, 505-519 (1959). - (2) Bapat, D. S., Subba Rao, B. C., Unni, M. K., and Venkataraman, K.: Tetrahedron Letters (1960), No. 5, 15 bis 19. - (3) Beckwith, R. S.: Austr. J. of Agric. Res. 6, 685-698 (1955). - (4) Broadbent, F. E., and Bradford, G, R.: Soil Sci. 74, 447-457 (1952). - (5) Brown, H. C., and Tierney, P. A.: J. Amer. Chem. Soc. 80, 1552-1558 (1958). - (6) Brown, H. C.: Hydroboration. W. A. Benjamin, New York (1962). - (7) Caglioti, L., Cainelli, G., und Selva, A.: Chim. Ind. (Milan) 
44, 36-37 (1962). - (8) Coffin, D. E., and De Long, W. A.: Trans. 7th Int. Congr. Soil Sci. II, 91-97 (1960). - (9) Deuel, H., und Dubach, P.: Helv. Chim. Acta 41, 1310-1321 (1958). - (10) Van Dijk, H.: Z. Pflanzenernähr., Düng., Bodenkunde 84, 150-155 (1959). (11) Van Dijk, H.: Sic. Proc. Roy. Dublin Soc. A 1, 163-176 (1960) - (12) Dubach, P., Mebta, N. C., und Deuel, H.: 102, 1-7 (1963), I. c. 10. - (13) Farmer, V. C., and Morrison, R. I.: 1. c. 11, 1, 85-104 (1960). - (14) Flaig, W., Scheffer, $F$., und Klamroth, B.: 1. c. 10, 71, 33-57 (1955). - (15) Flaig, W.: l. c. 8, B, $471 \rightarrow 478$ (1956). - (16) Forsyth, W. G. C.: J. Agr. Sci. 37, 132-138 (1947). - (17) Freudenberg, K.: Fortschr. Chem. Organ. Naturstoffe 20, 41-72 (1962). - (18) Fuchs, W.: Ges. Abhandl. Kenntnis Kohle 9, 176-181 (1930). - (19) Gillam, W. S.: 1. c. 4, 49, 433-453 (1940). - (20) Khanna, S. S., and Stevenson, F. J.: 1. c. 4, 93, 298-305 (1962). - (21) Kononowa, M. M.: Die Humusstoffe des Bodens. DVW, Berlin (1958). - (22) Van Krevelen, D. W., and Schuyer, J.: Coal Science. Elsevier, Amsterdam (1957). - (23) Kumada, K., and Aizawa, K.: Soil and Plant Food (Japan) 3, 152-159 (1958). - (24) Lewis, T. E., and Broadbent, F. E.: 1, c. 4, 91, 393-399 (1961). - (25) Martin, R. E., and Jay, R. R.: Anal. Chem. 34, 1007-1009 (1962). - (26) Martin, A. E., and Reeve, R.: J. Soil Sci. 9, 89-100 (1958). - (27) Marton, J., Adlet, E., and Persson, K. J.: Acta Chem. Scand. 15, 384-392 (1961). - (28) Mebta, N. C., Dubach, P., und Deuel, H.: Untersuchungen über die Molekulargewichtsverteilung von Huminstoffen durch Gelfiltration an Sephadex. 1. c. 10, im Druck. - (29) Meyer, W.: Die Bestimmung funktioneller Gruppen an Huminsubstanzen aus Böden. Diss. E.T.H. (1962). - (30) Pommer, A. M., and Breger, I. A: Geochim. Cosmoch. Acta 20, 30-44 (1960). - (31) Scheffer, F., und Ulrich, B.: Humus und Humusdüngung. Enke, Stuttgart (1960). - (32) Schnitzer, M., Shearer, D. A., and Wright, J. R.i 1. c. 4, 87, 252-257 (1959). - (33) Stadnikoff, G.: Kolloid-Z. 80, 60-68 (1937). - (34) Wright, G. F.: Organometallic Compounds for the Determination of Active Hydrogen. Inorganic Analysis 1, 155-195. Interscience, New York (1953). - (35) Wright, J. R., and Schnitzer, M.: Nature 184, 1462-1463 (1959). - (36) Wright, J. R., and Schnitzer, M.: l. c. 8, II, 120-127 (1960). - (37) Zetsche, F., and Reinhart, H.: Brennstoff Chem. 20, 84-87 (1939).

[2966] 